\title{
Effets aigus d'une irradiation corps entier sur la sécrétion pancréatique exocrine chez le porc
}

\author{
P. MONTI ${ }^{1}$, P. SCANFF ${ }^{1}$. C. JOUBERT ${ }^{1}$. M. VERGNET ${ }^{1}$, \\ S. GRISON ${ }^{1}$. N. GRIFFITHS
}

(Manuscrit resule 14 arril 2003, acespté le 31 octobre 2003 )

RÉSUMÉ Les effets radio-induits sur le pancréas, communément décrits chez les manmifères, concernent essentiellement des modifications structurales observées à long terme. En revanche, peu d'études traitent des conséquences fonctionnelles d'une irradiation sur le pancréas exocrine. Le but de ce travail est de suivre les effets aigus d'une irradiation corps entier sur le volume et l'activité enzymatique du suc pancréatique. Une irradiation gamma corps entier à la dose de 6 Gy a été réalisée chez des porcs. Une collecte de suc pancréatique, par cathétérisme permanent du canal pancréatique, a été réalisée avant et après exposition avec mesure en continu du volume sécrété. Sur chaqué échantillon de suc pancréatique, la concentration protéique ainsi que l'activité enzymatique de la trypsine, chymotrypsine, élastase, lipase et amylase ont été déterminées. Le volume de suc pancréatique sécrété quotidiennement diminue dès le lendemain de l'irradiation et le niveau sécrétoire ne revient jamais à des valeurs enregistrées avant irradiation. La quantité de protéines et les activités totales des enzymes sécrétées dans le suc pancréatique sont réduites de manière parallèle. Enfin, seules les activités spécifiques de l'élastase et de la lipase sont modifiées après l'irradiation. Ainsi, les conséquences d'une irradiation corps entier chez le porc se traduisent par une diminution importante et rapide de la sécrétion pancréatique exocrine, affectant à la fois les composantes hydrobicarbonatée et enzymatique. Ce phénomène pourrait contribuer aux perturbations de la sphère intestinale lors du syndrome aigu d'irradiation mais également générer des effets à plus long terme.

ABSTRACT Acute effects of whole body gamma irradiation on exocrine pancreatic secretion in the pig.

Reports on radiation damage to the pancreas deal essentially with long-term morphological changes with few data on pancreatic exocrine function. The aim of this work was to study the acute effects of whole body irradiation on volume and enzyme activities in the pancreatic juice. A whole body gamma irradiation ( 6 Gy) was investigated in pigs with continuous sampling of pancreatic juice before and after exposure via an indwelling catheter in the pancreatic duct. For each sample collected, total protein concentration and enzyme activities of trypsin, chymot rypsin, elastase, lipase and amylase were determined. Pancreatic juice volume was monitored during all periods of collection. The volume of pancreatic juice secreted daily decreased one day after irradiation and remained lower than the control values over the experimental period. Total proteins secreted in the pancreatic juice and total activities of pancreatic enzymes were reduced similarly. On the other hand,

\footnotetext{
1 IRSN, DPRH/SRBE. B.P. 17. 92262 Fontenaly-atux-Rones Codex. France.
} 
only specific activities of elastase and lipase were affected by irradiation. Whole body gamma irradiation resulted in a rapid and marked decrease of exocrine pancreatic secretion, in terms of volume as well as secreted enzymes. This may contribute in part to the intestinal manifestations of the acute and/or late radiation syndrome.

\section{Introduction}

Les conséquences d'une exposition partielle ou totale à fortes doses d'irradiation (> à $10 \mathrm{~Gy}$ ) conduisent à des perturbations fonctionnelles le long du tractus digestif. Ces dysfonctionnements peuvent avoir des conséquences importantes dans la mise en place des pathologies mixtes radio-induites impliquant des atteintes hématopoiétique, cutanée, digestive et pulmonaire (Becciolini et al., 1974 ; Frankendal, 1974).

Parmi les différentes fonctions du tractus digestif, les sécrétions exocrines telles que les sécrétions gastrique, pancréatique et biliaire sont susceptibles d'être modifiées après irradiation. Le rôle du pancréas exocrine dans la physiologie digestive est particulièrement important puisqu'il permet l'hydrolyse des différents nutriments qui arrivent dans la lumière digestive (glucides, protides et lipides), afin qu'ils soient assimilables par l'organisme. Pour cela, diverses enzymes (protéases, lipase et amylase) sont sécrétées dans le suc pancréatique, en réponse à des stimuli alimentaires principalement, et leur sécrétion dépend d'un contrôle neuro-hormonal. Les effets de l'irradiation sur la sécrétion pancréatique exocrine restent peu décrits et les études portent principalement sur les modifications structurales du pancréas à des temps tardifs après irradiation (Volk et Wellmann, 1968 ; Du Toit et al., 1987 ; Ahmadu-Suka et al., 1988). Cependant, l'implication de la sécrétion pancréatique exocrine dans la mise en place du syndrome aigu d'irradiation a été plusieurs fois avancée. Généralement, pour des fortes doses d'exposition, l'hypothèse retenue repose sur l'effet agressif du suc pancréatique qui serait d'autant plus important que la muqueuse intestinale peut déjà être endommagée par l'irradiation. Archambeau et al. (1966) et Morgenstern et al. (1970) ont ainsi démontré que la ligature du canal pancréatique, permettant l'exclusion des enzymes pancréatiques de la lumière intestinale, prolongeait la survie de 7 jours de chiens irradiés dans la région abdominale. D'autres études ont montré que la composition des contenus luminaux pouvait contribuer au développement des effets radio-induits sur le système digestif (Hiatt et Warner, 1967 ; Mulholland et al., 1984). Par exemple, l'absence totale de contenus intestinaux, de bile uniquement ou de suc pancréatique au moment de l'irradiation, permet de réduire de façon importante les altérations morphologiques de l'intestin. Cinq jours après l'irradiation d'une anse intestinale aux rayons $X$ ( 11 Gy), différents paramètres structuraux tels que la cellularité dans la crypte, le nombre de cellules à mucus et l'épaisseur de la muqueuse sont améliorés dans les segments 
où des agents potentiellement agressifs, acides biliaires ou protéases, ont été inactivés (Delaney et Bonzak, 1992). Plusieurs études vont en faveur de l'importance de l'effet délétère de la sécrétion pancréatique dans la réponse radioinduite de l'intestin (Morgenstern et Hiatt. 1967 ; Patin et al., 1970), les enzymes protéolytiques étant principalement impliquées (Rachootin et al., 1972 ; HauerJensen et al., 1995). Ainsi, Palladino et al. (1982) ont montré que l'injection par voie intra-veineuse d'inhibiteurs protéasiques avant l'irradiation permettait d'augmenter la survie de poulet ou de souris irradiés. L'implication de la sécrétion pancréatique exocrine dans les altérations radio-induites du tractus digestif s'appuie également sur le fait qu'une baisse importante de l'absorption intestinale des nutriments a été démontrée après irradiation (Thomson et al., 1983). Cet effet dépressif serait en partie dû à une diminution du potentiel enzymatique présent dans la lumière intestinale, principalement représenté par les enzymes pancréatiques.

Ainsi, nous avons étudié chez le porc les effets précoces d'une irradiation corps entier à la dose de 6 Gy sur la sécrétion pancréatique exocrine. La dose de 6 Gy a été choisie pour se placer dans des conditions d'atteinte fonctionnelle au niveau du tractus gastro-intestinal, sans pour autant engendrer le syndrome gastro-intestinal proprement dit. Le porc représente un modèle expérimental pertinent du fait de la similitude de son système digestif avec celui de l'homme (Stevens, 1977), de la mise en place d'un cathétérisme permanent dans le canal pancréatique permettant la collecte d'échantillons pendant plusieurs jours, et enfin un volume corporel permettant une dosimétrie comparative à celle de l'homme. Un suivi quotidien et en continu, du volume et des activités enzymatiques du suc pancréatique, a été réalisé avant et après exposition, afin de pouvoir suivre la mise en place de la réponse radio-induite de la sécrétion pancréatique exocrine.

\section{Matériels et méthodes}

\subsection{Animaux et alimentation}

Cinq porcs castrés (Large White croisé Landrace) âgés de 4 à 6 mois ont été utilisés. Les animaux pesaient environ $60 \mathrm{~kg}$ au début de l'expérimentation, puis leur poids a été mesuré le jour de l'irradiation et en fin de période expérimentale. Pendant toute l'expérimentation, les animaux sont maintenus en contention dans des cages à métabolisme, et sont nourris avec une alimentation standard (protéines : $15 \%$; lipides : $3 \%$; amidon : $57 \%$ et cellulose : $6 \%$, exprimés en \% de l'aliment - énergie brute $=3762 \mathrm{kcal} / \mathrm{kg})$ à raison de deux repas par jour $(9 \mathrm{~h} 00$ et $16 \mathrm{~h} 00$ ) de $800 \mathrm{~g}$ chacun, et reçoivent de l'eau ad libitum entre les repas. 


\subsection{Chirurgie}

La mise en place du cathétérisme pancréatique permanent et d'une ré-introduction duodénale a été pratiquée chez les animaux, dix jours avant le début de l'expérimentation, selon la méthode de Corring et al. (1972). À jeun depuis la veille au soir, les porcs sont anesthésiés au masque avec de l'halothane $(2,5-3,5 \%)$ après une sédation à la Kétamine $(8 \mathrm{mg} / \mathrm{kg}, \mathrm{i} . \mathrm{m}$. $)$. La mise en place d'un cathéter en polyéthylène est pratiquée dans le canal pancréatique. Un $2^{\mathrm{e}}$ cathéter est inséré dans le duodénum, proche du site naturel d'arrivée du canal pancréatique. Les deux cathéters sont extériorisés par le flanc droit de l'animal.

Dans un second temps, les animaux sont munis d'un cathéter permanent dans la veine jugulaire et dans l'artère carotide gauches afin d'une part, de pouvoir obtenir des échantillons sanguins, et d'autre part d'avoir un abord veineux pour les supports thérapeutiques. Un traitement antibiotique (Bipénicilline, $10^{6} \mathrm{U} /$ jour) est administré au cours de l'intervention chirurgicale dans la cavité péritonéale puis pendant 3 jours après la chirurgie par voie veineuse. Une période de récupération est ménagée sur 10 jours au cours de laquelle les animaux récupèrent progressivement le niveau normal de leur prise alimentaire.

\subsection{Schéma expérimental}

Les cathéters permettant la collecte de suc pancréatique ainsi que sa réintroduction duodénale sont connectés à un appareillage automatique. Ce montage permet de ré-infuser le suc pancréatique dès son extériorisation, tout en mesurant le volume sécrété (Juste et al., 1983). Au cours de la période de récupération post-opératoire, la totalité de la sécrétion pancréatique est réintroduite à l'animal alors que pendant la phase expérimentale, un échantillon représentant 4 à $8 \%$ de la sécrétion totale est dérivé en continu. Chez tous les animaux, une $1^{\text {re }}$ période expérimentale de 3 jours est réalisée avant l'irradiation et dès le lendemain de l'irradiation, une $2^{\mathrm{e}}$ période expérimentale se poursuit sur 5 jours consécutifs. Chaque animal reste ainsi son propre témoin.

Un collecteur de fractions permet d'obtenir des échantillons horaires de suc pancréatique à partir desquels un pool représentant la sécrétion écoulée sur 24 heures a été constitué.

Les échantillons de suc pancréatique ainsi conditionnés ont été stockés à $-20^{\circ} \mathrm{C}$ jusqu'à leur analyse.

Les animaux sont suivis quotidiennement et les manifestations cliniques pouvant apparaître après irradiation sont contrôlées. Ainsi, la prise alimentaire, 
la température corporelle, l'apparition de vomissement et de diarrhée sont particulièrement surveillées. Lorsque la consommation alimentaire diminue de plus de $50 \%$ par rapport à la ration normale, les animaux sont perfusés avec une solution de glucose $5 \%$ et de $\mathrm{NaCl}(0,9 \%)$ à raison de $250 \mathrm{ml}$ de chaque par jour. De la même façon, en cas d'hyperthermie sévère et maintenue, un traitement d'antibiotiques (Bipénicilline, $10^{6} \mathrm{U} /$ jour) est administré par voie intraveineuse.

À partir du $6^{\mathrm{e}}$ jour, l'état clinique des animaux s'est sévèrement détérioré (hyperthermie, anorexie, apathie), et ne nous a plus permis de poursuivre l'expérimentation dans des conditions satisfaisantes, pour des raisons éthiques d'une part, et du fait d'un tarissement quasi complet de la sécrétion pancréatique exocrine d'autre part. Tous les animaux ont été euthanasiés le $7^{\mathrm{e}}$ jour après irradiation, par surdosage d'agent anesthésique (Halothane).

Les expérimentations ont été menées en accord avec la législation française concernant l'expérimentation animale (Ministère de l'agriculture, acte $\mathbf{n}^{\circ} 87-848$ du 19 octobre 1987).

\subsection{Procédures d'irradiation des animaux}

À jeun depuis la veille au soir, les porcs ont été exposés à une irradiation gamma corps entier à la dose de $6 \mathrm{~Gy}\left({ }^{60} \mathrm{Co}\right.$, débit : $0,5 \mathrm{~Gy} / \mathrm{min}$ au plan médian). L'irradiateur est constitué de 8 sources individuelles de ${ }^{60} \mathrm{Co}$ ( 4 de chaque côté) d'une activité totale d'environ $2,2 \times 10^{14} \mathrm{~Bq}$. Avant l'irradiation, les animaux sont légèrement anesthésiés avec une injection de Kétamine $(8-10 \mathrm{mg} / \mathrm{kg}, \mathrm{i} . \mathrm{m}$.) puis placés dans des hamacs afin d'obtenir une irradiation la plus homogène possible. Avant le début de la période expérimentale, les animaux ont subi une «sham irradiation » (irradiation fictive sans sortie des sources), afin de vérifier les effets possibles du jeûne et de la sédation par la Kétamine sur les différents paramètres observés.

\subsection{Analyse des échantillons de suc pancréatique}

Les protéines totales sécrétées dans le suc pancréatique sont déterminées selon la méthode de Bradford (1976). Après activation avec de l'entérokinase ou de la trypsine, les activités des enzymes protéolytiques (trypsine, chymotrypsine et élastase) ont été déterminées par méthodes colorimétriques (Bieth et al., 1966, 1968, 1974 respectivement). Les activités lipasique et amylasique ont également été mesurées dans chaque échantillon. Les résultats sont exprimés en activité totale d'enzyme sécrétée dans le suc pancréatique (unité arbitraire d'activité $\times$ volume de suc pancréatique sécrété) ainsi qu'en activité spécifique (activité/mg protéines totales sécrétées). 


\subsection{Analyse statistique}

La moyenne des valeurs obtenues pendant les 3 jours de la $1^{\text {re }}$ période expérimentale a été utilisée pour représentée la valeur témoin de chaque porc. Toutes les valeurs sont exprimées en moyenne \pm écart type à la moyenne et les résultats ont été analysés à l'aide d'un test de student pairé (*p $<0,05 ; * * \mathrm{p}<0,01$; $* * * \mathrm{p}<0,001)$.

\section{Résultats}

\subsection{Animaux et consommation alimentaire}

Diverses manifestations cliniques ont été notées au cours de la période postirradiation. Des vomissements sont apparus chez tous les animaux au cours de la $1^{\text {re }}$ heure qui a suivi l'exposition. En revanche, aucun épisode diarrhéique n'a été observé pendant toute la période expérimentale. Une température corporelle normale $\left(38{ }^{\circ} \mathrm{C}\right) \mathrm{s}^{\prime}$ est maintenue jusqu' au $4^{\mathrm{e}}$ jour après irradiation, et a ensuite augmenté de manière importante $\left(>40^{\circ} \mathrm{C}\right)$, nécessitant une administration d'antibiotiques par voie veineuse.

Dans tous les cas, une phase de prostration a été observée dans les heures qui ont suivi l'irradiation, puis les animaux se sont comportés et alimentés de façon normale jusqu'au $3^{\mathrm{e}}$ jour après l'exposition. À partir de là, une baisse de la consommation alimentaire a été enregistrée $(-44 \%, \mathrm{p}<0,05)$, et s'est confirmée jusqu'à la fin de la période expérimentale (Fig. 1) où la prise alimentaire ne représente plus que le tiers de sa valeur avant irradiation. De façon concomitante, les animaux ont montré une perte de poids d'environ $1 \mathrm{~kg}$ /jour alors qu'en période témoin, le gain de poids moyen journalier était de $0,8 \mathrm{~kg}$. Au $6^{\mathrm{e}}$ jour après irradiation, la majorité des animaux présentaient un état d'apathie important, nécessitant une perfusion d'une solution de glucose- $\mathrm{NaCl}$ deux fois par jour. Tous les animaux ont été euthanasiés le $7^{\mathrm{e}}$ jour pour les raisons citées précédemment.

\subsection{Volume et protéines totales du suc pancréatique}

Le volume de suc pancréatique sécrété par 24 heures est de $3700 \pm 250 \mathrm{ml}$ en période témoin. Dès le lendemain de l'irradiation, la sécrétion de suc pancréatique chute de $40 \%(\mathrm{p}<0,001)$ et cette réduction se poursuit jusqu'au $3^{\mathrm{e}}$ jour après l'exposition (Fig. 2). À la fin de l'expérimentation, le volume de la sécrétion pancréatique ne représente plus que $30 \%(\mathrm{p}<0,01)$ de la valeur mesurée avant irradiation. La mesure du volume a également été enregistrée sur différentes 


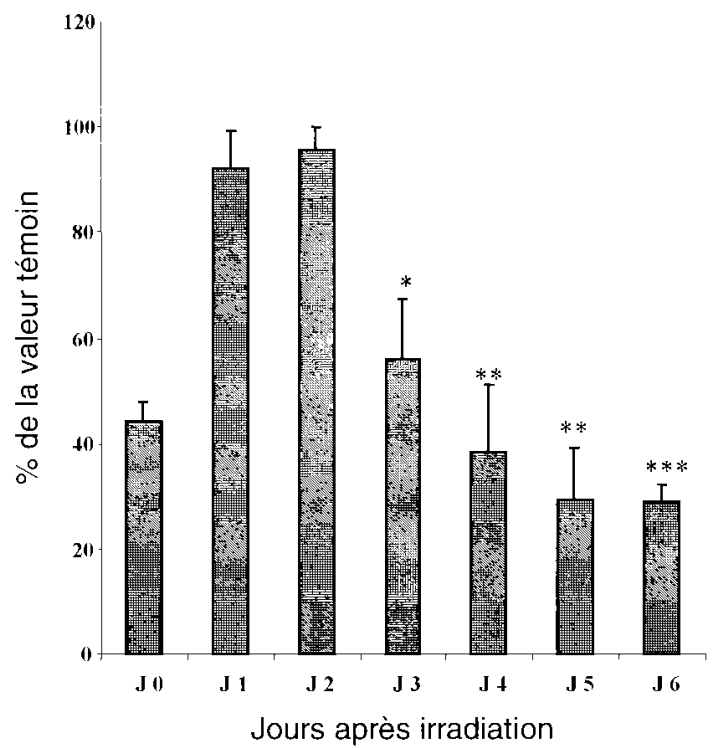

Figure 1 - Évolution de la prise alimentaire de porcs irradiés corps entier à la dose de 6 Gy. Les valeurs représentent la moyenne \pm écart-type à la moyenne obtenue avec 5 porcs, et les résultats sont exprimés en pourcentage des valeurs obtenues pendant les 3 jours de la période avant irradiation (période témoin). Les données sont analysées avec un test-t pairé de Student pour comparer les valeurs mesurées après irradiation à celle représentant la valeur témoin $\left({ }^{*} p<0,05 ;{ }^{* *} p<0,01 ;{ }^{* * *} p<0,001\right)$.

Effect of 6 Gy total body irradiation on daily food intake of pigs. Values are mean $\pm S E M$ of 5 pigs and data are expressed as a percentage of average of values measured on 3 days before irradiation (control period). Statistical comparison was made with a paired student's t-test $(* p<0.05 ; * * * 0<0.01 ; * * * * 0<0.001)$.

périodes du nycthémère : périodes post-prandiales ( 4 heures qui suivent les repas), inter-digestive (entre les deux repas) et basale (période nocturne). Cela a permis de préciser que cette réduction du volume observée sur 24 heures provient essentiellement d'une baisse en périodes post-prandiales (données non représentées).

Par ailleurs, la concentration protéique dans le suc pancréatique mesurée sur 24 heures n'a pas été altérée par l'irradiation (Fig. 3). En conséquence, la quantité de protéines suit les variations du volume de suc pancréatique sécrété (Fig. 3), et diminue de façon significative à partir du $3^{\mathrm{e}}$ jour après l'irradiation, ne représentant plus que $28 \%(\mathrm{p}<0,01)$ de la valeur témoin à la fin de l'expérimentation. 


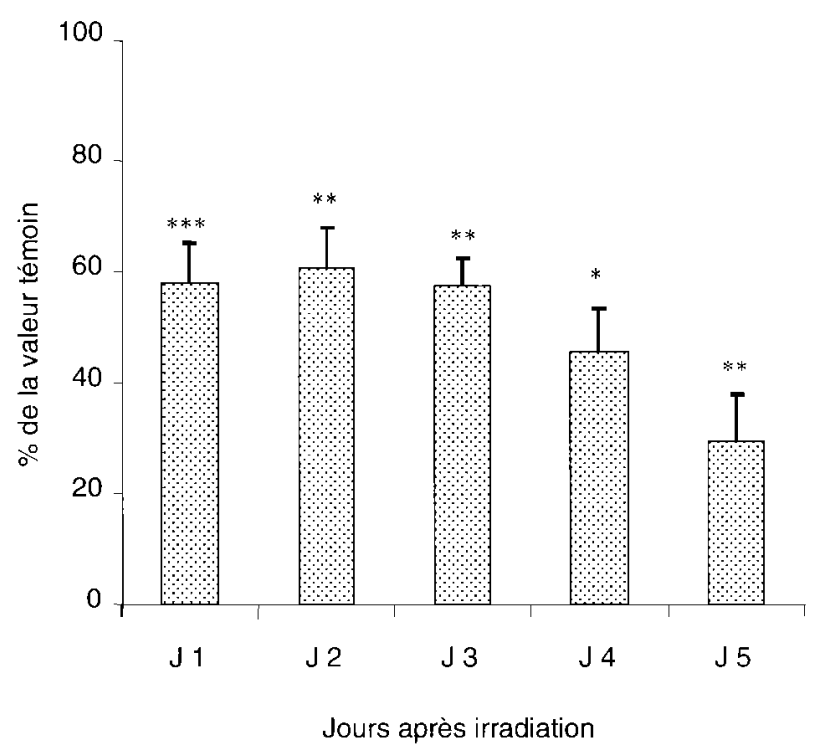

Figure 2-Effet d'une irradiation corps entier de 6 Gy sur le volume de suc pancréatique sécrété par 24 heures. Les valeurs représentent la moyenne \pm écart-type à la moyenne obtenue avec 5 porcs, et les résultats sont exprimés en pourcentage des valeurs obtenues pendant les 3 jours de la période avant irradiation (période témoin). Les données sont analysées avec un test-t pairé de Student pour comparer les valeurs mesurées après irradiation à celle représentant la valeur témoin $(* p<0,05 ; * * p<0,01 ; * * * p<0,001)$.

Effect of 6 Gy total body irradiation on the volume of the pancreatic juice secreted daily. Values are mean \pm SEM of 5 pigs and data are expressed as a percentage of average of values measured on 3 days before irradiation (control period). Statistical comparison was made with a Paired student's t-test $(* p<0.05 ; * *<0.01 ; * * * p<0.001)$.

\subsection{Activités totales des enzymes pancréatiques}

La mesure de l'activité totale des différentes enzymes pancréatiques, faite sur l'échantillon représentant la sécrétion écoulée sur 24 heures, montre que la réponse à l'irradiation se cale sur les modifications observées au niveau du volume de suc pancréatique sécrété.

\subsubsection{Enzymes protéolytiques}

L'activité totale de la trypsine, de la chymotrypsine et de l'élastase sont toutes modifiées par l'irradiation (Fig. 4). Pour la trypsine, l'activité totale sécrétée quotidiennement est faible dès le lendemain de l'irradiation, et cette diminution reste constante pendant au moins deux jours. À la fin de la période expérimentale, 


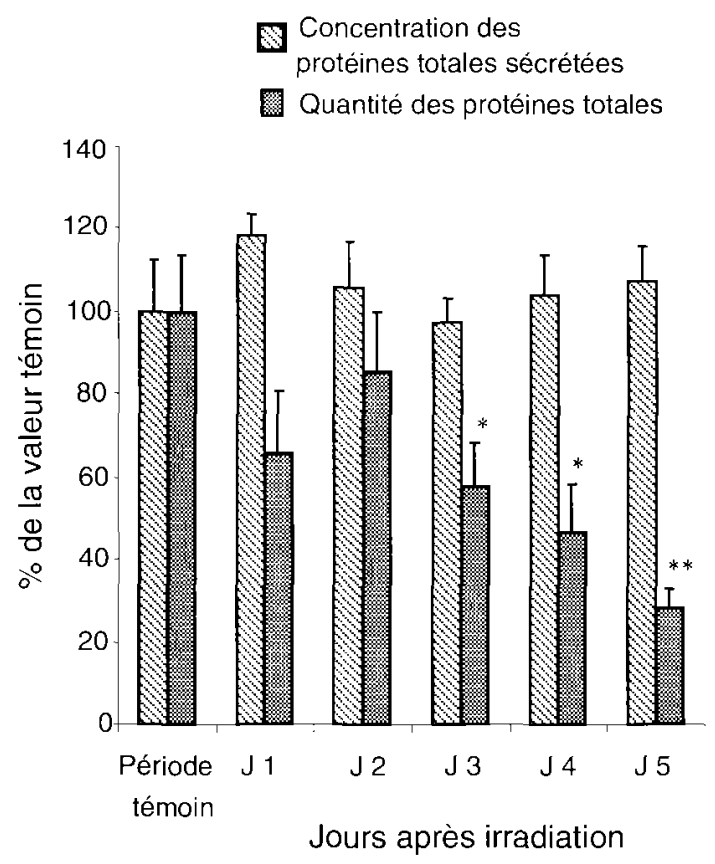

Figure 3 - Concentration protéique et quantité des protéines totales sécrétées dans le suc pancréatique chez des porcs après une irradiation corps entier de 6 Gy. Les valeurs représentent la moyenne \pm écart-type à la moyenne obtenue avec 5 porcs, et les résultats sont exprimés en pourcentage des valeurs obtenues pendant les 3 jours de la période avant irradiation (période témoin). Les données sont analysées avec un test-t pairé de Student pour comparer les valeurs mesurées après irradiation à celle représentant la valeur témoin $\left({ }^{*} p<0,05 ; * * p<0,01\right)$.

Concentration and amount of total protein secreted daily in the pancreatic juice of pigs after 6 Gy total body irradiation. Values are mean \pm SEM of 5 pigs and data are expressed as a percentage of average of values measured on 3 days before irradiation (control period). Statistical comparison was made with a paired student's t-test $(* p<0.05 ; * * p<0.0 I)$.

l'activité trypsique du suc pancréatique qui arrive au niveau du duodénum ne représente plus que $1 / 5$ de celle mesurée avant irradiation $(p<0,05)$. Pour la chymotrypsine, une baisse de $30 \%$ est observée à partir du $3^{\mathrm{e}}$ jour $(\mathrm{p}<0,05)$ et s'amplifie progressivement pour atteindre $65 \%$ le $5^{\mathrm{e}}$ jour $(\mathrm{p}<0,05)$. Enfin, l'activité de l'élastase est la plus sévèrement affectée puisque dès le lendemain de l'irradiation, sa valeur est réduite de moitié $(\mathrm{p}<0,05)$; cette réponse se prolonge et s'intensifie jusqu'au dernier jour de l'expérimentation où elle ne représente plus que $15 \%$ de la valeur témoin $(\mathrm{p}<0,01)$. 


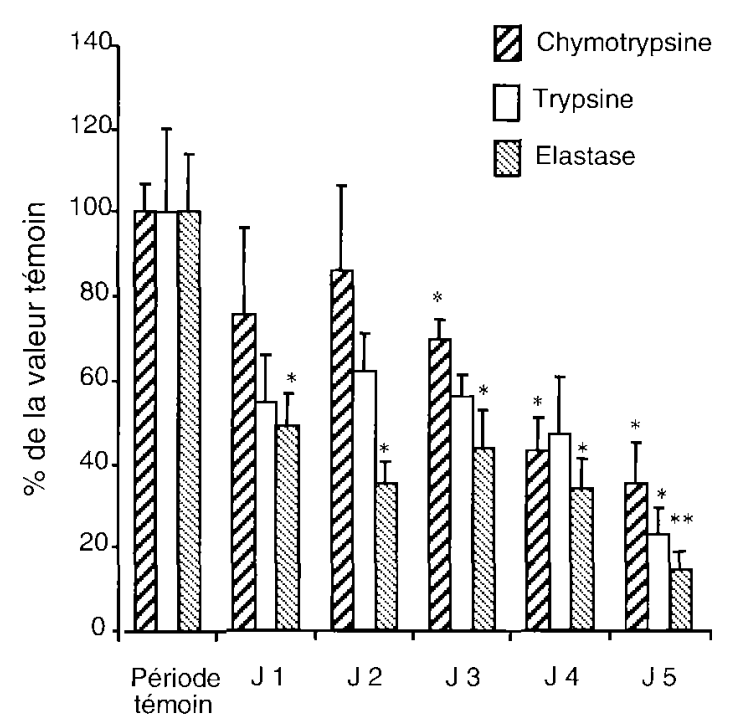

Jours après irradiation

Figure 4-Évolution de l'activité totale des enzymes protéolytiques mesurée sur 24 heures dans le suc pancréatique de porcs après une irradiation corps entier de $6 G y$. Les valeurs représentent la moyenne \pm écart-type à la moyenne obtenue avec 5 porcs, et les résultats sont exprimés en pourcentage des valeurs obtenues pendant les 3 jours de la période avant irradiation (période témoin). Les données sont analysées avec un test-t pairé de Student pour comparer les valeurs mesurées après irradiation à celle représentant la valeur témoin $\left({ }^{*} p<0,05 ;{ }^{*} p<0,0 I\right)$.

Time course of total activity of proteolytic enzymes secreted daily in the pancreatic juice of pigs following 6 Gy total body irradiation. Values are mean \pm SEM of 5 pigs and data are expressed as a percentage of average of values measured on 3 days before irradiation (control period). Statistical comparison was made with a Paired student's $t$-test $(* p<0.05 ; * * p<0.01)$.

\subsubsection{Amylase et lipase}

Les activités totales de l'amylase et de la lipase sécrétées quotidiennement dans le suc pancréatique montrent également une forte baisse radio-induite (Fig. 5). Cet effet est significatif pendant toute la période après irradiation; ainsi, à la fin de l'expérimentation, les activités amylasique et lipasique ne représentent plus que 30 et $20 \%$ de la valeur mesurée avant l'exposition aux rayonnements ionisants ( $\mathrm{p}<0,01$ et $\mathrm{p}<0,05$ respectivement).

\subsection{Activité spécifique des enzymes pancréatiques}

Les valeurs des activités spécifiques des différentes enzymes pancréatiques mesurées avant et après irradiation sont représentées dans le tableau I. L'évolution 


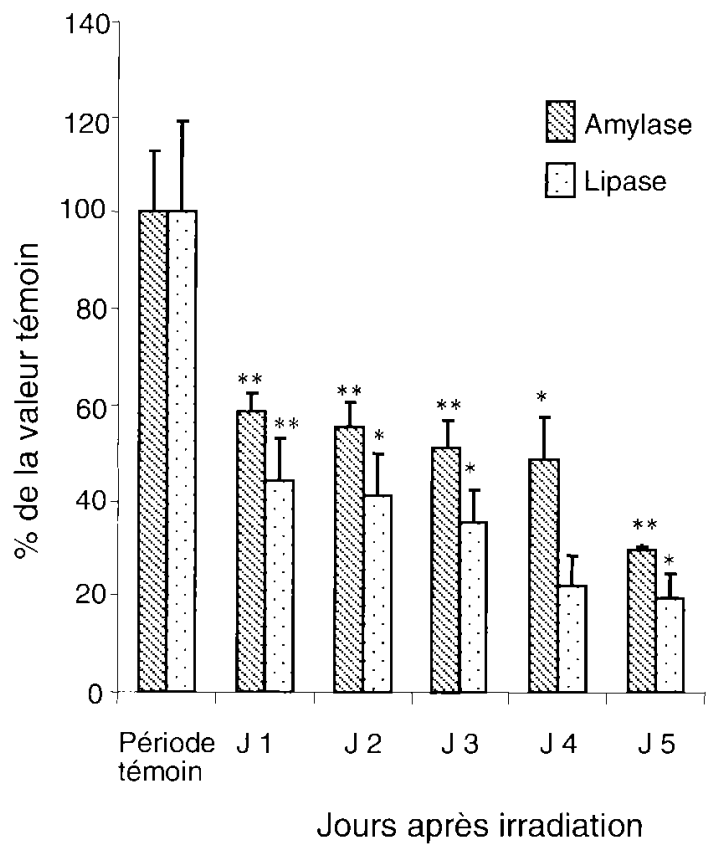

Figure 5-Évolution de l'activité totale de l'amylase et de la lipase mesurée sur 24 heures dans le suc pancréatique de porcs après une irradiation corps entier de 6 Gy. Les valeurs représentent la moyenne \pm écart-type à la moyenne obtenue avec 5 porcs, et les résultats sont exprimés en pourcentage des valeurs obtenues pendant les 3 jours de la période avant irradiation (période témoin). Les données sont analysées avec un test-t pairé de Student pour comparer les valeurs mesurées après irradiation à celle représentant la valeur témoin $\left({ }^{*} p<0,05 ; *^{*} p<0,01\right)$.

Time course of total activity of amylase and lipase secreted daily in the pancreatic juice of pigs following 6 Gy total body irradiation. Values are mean $\pm S E M$ of 5 pigs and data are expressed as a percentage of the average of values measured on 3 days before irradiation (control period). Statistical comparison was made with a Paired student's t-test $(* p<0.05 ; * * p<0.01$ ).

générale de ces activités met en évidence un comportement différent des enzymes protéolytiques d'une part, et de l'amylase et de la lipase d'autre part.

\subsubsection{Enzymes protéolytiques}

Les activités spécifiques de la trypsine et de la chymotrypsine ne sont pas affectées par l'irradiation. En revanche, seule l'activité de l'élastase présente une variation radio-induite avec une baisse significative qui atteint $48 \%$ le $5^{\mathrm{e}}$ jour après l'exposition. 


\section{TABLEAU I}

Activités spécifiques des enzymes pancréatiques sécrétées quotidiennement dans le suc pancréatique de porcs après une irradiation corps entier de 6 Gy. Les valeurs représentent la moyenne \pm écart-type à la moyenne obtenue avec 5 pores, et les résultats sont exprimés en unités

arbitraires/mg de protéines totales sécrétées dans le suc pancréatique. La valeur têmoin représente la moyenne des valeurs obtenues pendant les 3 jours de la période avant irradiation. Les données sont analysées avec un test-t pairé de Student pour comparer les valeurs mesurées après irradiation à celle représentant la valeur témoin $\left({ }^{*} \mathbf{p}<0,05\right)$.

Specific activities of pancreatic enzymes secreted daily in the pancreatic juice of pigs after $6 \mathrm{~Gy}$ total body irradiation. Values are mean \pm SEM of 5 pigs and data are expressed in arbitrary units/mg of total proteins secreted in the pancreatic juice. Control value represented the average of values measured on 3 days before irradiation (control period). Statistical comparison was made with a Paired student's t-test $\left({ }^{*} p<0.05\right)$.

\begin{tabular}{|c|c|c|c|c|c|}
\hline 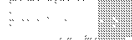 & Trypine & Chymotypuine & Elaxtase & Amylase & Lipase \\
\hline $\begin{array}{l}\text { période } \\
\text { lémoin }\end{array}$ & $\begin{array}{c}4,10 \\
(0.34)\end{array}$ & $\begin{array}{r}144,5 \\
(15,5)\end{array}$ & $\begin{array}{l}\mathbf{5 2 2 , 0} \\
(86,5)\end{array}$ & $\begin{array}{c}39385 \\
(858)\end{array}$ & $\begin{array}{l}160,6 \\
(7,6)\end{array}$ \\
\hline J1 & $\begin{array}{c}\mathbf{4 , 1 3} \\
(0,37)\end{array}$ & $\begin{array}{l}\mathbf{1 4 4}, \mathbf{7} \\
(13,7)\end{array}$ & $\begin{array}{l}\mathbf{3 5 9}, \mathbf{6} \\
(32,4)\end{array}$ & $\begin{array}{l}41290 \\
(6087)\end{array}$ & $\begin{array}{c}129,7^{*} \\
(9,0)\end{array}$ \\
\hline $\mathbf{J} 2$ & $\begin{array}{c}3,85 \\
(0.47)\end{array}$ & $\begin{array}{l}\mathbf{1 6 7 , 2} \\
(14,2)\end{array}$ & $\begin{array}{l}\mathbf{2 6 9 , 3} \\
(32,6)\end{array}$ & $\begin{array}{l}\mathbf{3 6} \mathbf{5 3 7} \\
(5393)\end{array}$ & $\begin{array}{c}111,0^{*} \\
(5,6)\end{array}$ \\
\hline $\mathbf{J 3}$ & $\begin{array}{c}3,78 \\
(0,36)\end{array}$ & $\begin{array}{l}\mathbf{1 6 8 , 7} \\
(20,4)\end{array}$ & $\begin{array}{l}344,5 \\
(41,3)\end{array}$ & $\begin{array}{l}37632 \\
(2571)\end{array}$ & $\begin{array}{c}111,7^{*} \\
(11,8)\end{array}$ \\
\hline $\mathrm{J} 4$ & $\begin{array}{c}3,14 \\
(0,53)\end{array}$ & $\begin{array}{l}163,7 \\
(17,0)\end{array}$ & $\begin{array}{r}\mathbf{3 4 3 , 2} \\
(44.8)\end{array}$ & $\begin{array}{r}43910 \\
(1 \quad 833)\end{array}$ & $\begin{array}{l}90,7^{*} \\
(4.9)\end{array}$ \\
\hline $\mathrm{J5}$ & $\begin{array}{c}3,09 \\
(0.62)\end{array}$ & $\begin{array}{l}159,7 \\
(26,4)\end{array}$ & $\begin{array}{c}273,2^{*} \\
(39,3)\end{array}$ & $\begin{array}{l}41071 \\
(3744)\end{array}$ & $\begin{array}{l}\mathbf{1 1 2 , 4} \\
(19,4)\end{array}$ \\
\hline
\end{tabular}

\subsubsection{Lipase et amylase}

Les activités spécifiques de la lipase et de l'amylase mesurées dans le suc pancréatique répondent différemment à l'irradiation ; ainsi, pour l'activité lipasique, une baisse de $20 \%$ est observée dès le lendemain de l'exposition ( $\mathrm{p}<0,05$ ) et persiste jusqu'au dernier jour de l'expérimentation. L'activité spécifique de l'amylase n'est quant à elle jamais altérée par l'irradiation.

On retient donc que ces variations d'activités spécifiques reflètent une modification qualitative modérée de la sécrétion de suc pancréatique déversé dans le duodénum après irradiation.

\section{Discussion}

La symptomatologie du syndrome gastro-intestinal chez le porc a été bien décrit pour des doses largement supérieures à 10 Gy (Daburon et al., 1972). La dose de $6 \mathrm{~Gy}$ au plan médian utilisée dans notre étude nous a permis de provoquer des modifications fonctionnelles sans pour autant engendrer le syndrome gastrointestinal, tel qu'il est classiquement décrit dans ce modèle animal. Concernant les 
manifestations cliniques, les vomissements ont représenté le symptôme observé chez tous les animaux, environ 30 minutes après l'exposition. Cet effet prodromal, observé également chez l'homme pour des doses identiques (Anno et al., 1989), n'a été cependant décrit que pour des doses supérieures, chez le porc adulte de race Pitman-Moore (Daburon et al., 1972), le chien (Dubois et al., 1984) et le primate non-humain (Middleton et Young, 1975). Par ailleurs, la dose de 6 Gy n'a en aucun cas permis d'observer un symptôme diarrhéique quelque soit le jour après l'exposition; il est fort probable que dans ces conditions d'irradiation, la dose délivrée à l'intestin ne soit pas suffisante pour engendrer une atteinte structurale majeure et/ou un déséquilibre important de la balance hydrominérale.

La baisse de la consommation alimentaire, qui atteint $70 \%$ dès le $5^{\mathrm{e}}$ jour après exposition est concomitante à la mise en place d'un état apathique sévère observé chez tous les animaux.

L'atteinte précoce des sécrétions pancréatiques exocrines vient s'ajouter aux diverses manifestations radio-induites observées au niveau du tractus digestif du porc. Ainsi, l'effet dépressif que nous avons observé sur l'activité pancréatique est concomitant avec l'inhibition de la vidange gastrique (Rémy et al., 1975), la baisse de l'absorption intestinale (Rémy et al., 1974) et des variations qualitatives de la sécrétion biliaire (Scanff et al., 1997). Des modifications du volume de suc pancréatique ont également été rapportées chez le porc irradié partiellement avec des doses d'exposition similaires (Corring et al., 1974). Dans notre étude, la récolte de suc pancréatique le jour de l'irradiation a été fortement réduite, en partie dû à certaines contraintes expérimentales, tels que le jeune de 24 heures précédent l'irradiation et l'anesthésie pratiquée pendant l'exposition. Après consommation du repas de 16 heures, soit environ 6 heures après l'exposition, le niveau sécrétoire de suc pancréatique est revenu à des valeurs normales (résultats non-montrés), montrant que la réponse post-prandiale de la sécrétion pancréatique est encore « opérationnelle » dans les heures qui suivent l'irradiation. L'étroite relation entre la quantité d'aliment ingérée et le niveau de la sécrétion pancréatique exocrine a été largement décrite chez diverses espèces. Ainsi, chez le porc, Corring et al. (1972) ont montré que cette réponse sécrétoire, visant à augmenter la quantité d'enzymes dans la lumière duodénale, pouvait à la fois résulter d'une augmentation du volume ou de la concentration en enzymes du suc pancréatique, ces deux composantes étant sous un contrôle neuro-hormonal bien spécifique. Dans notre étude, la baisse de la sécrétion pancréatique exocrine observée dès le lendemain de l'irradiation n'est cependant pas concomitante à une réduction de la consommation alimentaire, cette dernière ne débutant que 3 jours après l'exposition. Or, nous avons observé que c'est au cours des phases post-prandiales que la sécrétion pancréatique est la plus affectée, alors que la quantité ingérée n'est pas modifiée. Ces différents éléments vont en faveur d'une atteinte des voies de 
régulation de la sécrétion pancréatique, à la fois pour la composante hydrobicarbonatée et pour la composante enzymatique. Gudkova $(1957,1960)$ a montré chez le chien que la protection de la zone du pancréas pendant l'irradiation n'avait pas empêché l'apparition de désordres fonctionnels de la sécrétion pancréatique exocrine, identiques à ceux observés chez les animaux irradiés corps entier, mettant ainsi en avant les désordres radio-induits de la régulation du pancréas.

Les systèmes de régulation de la sécrétion pancréatique exocrine sont multiples et font intervenir à la fois une composante nerveuse et hormonale. La phase gastrique de cette régulation permet entre autre la libération d'acide gastrique ; ce dernier représente un puissant stimulant de la sécrétion pancréatique exocrine en régulant la sécrétion des bicarbonates via l'action de la sécrétine (Solomon, 1984). La libération de cette hormone est fortement corrélée à la concentration en ion $\mathrm{H}^{+}$ et donc à l'acidité mesurée au niveau de la lumière duodénale (Schaffalitzky de Muckadell et al., 1981). L'effet d'une irradiation corps entier sur la sécrétion gastrique a été étudié chez le porc (Rémy et al., 1975) et se traduit par une forte diminution de la sécrétion acide, durant 3 jours après l'exposition. Ainsi, il est probable que cette baisse de la libération d'acide chlorhydrique puisse engendrer une altération des taux plasmatiques de sécrétine, qui à son tour pourrait conduire à une diminution de la composante hydro-bicarbonatée du suc pancréatique. Pour appuyer cette hypothèse, Corring et al. (1974) ont montré que l'injection intraveineuse de sécrétine pratiquée chez des porcs 5 jours après une irradiation à la dose de $6 \mathrm{~Gy}$, se traduisait par une nette augmentation du volume de suc pancréatique. Ces résultats montrent que, même à un moment où le niveau sécrétoire est le plus affaibli par l'irradiation, le pancréas conserve sa capacité sécrétoire en réponse à des stimulations physiologiques, mais n'est cependant plus capable de l'exprimer spontanément. Une altération de la régulation du pancréas exocrine semble donc être impliquée dans la réponse radio-induite de cet organe. Le même type de conclusion a été proposé pour la réponse de la sécrétion gastrique à l'irradiation qui serait imputable à des défauts de régulation plus qu'à l'action directe des rayonnements sur l'estomac (Rémy et al., 1975).

Si l'on considère que l'activité spécifique d'une enzyme reflète son « indice de pureté » au sein du mélange, la mesure de cette activité permet d'apprécier en quelque sorte la composition relative en enzymes. Nous avons constaté que nos conditions d'irradiation n'ont pas engendré de variations qualitatives notoires du suc pancréatique qui arrive dans la lumière duodénale. À l'inverse, les modifications quantitatives des taux d'enzymes sécrétées sont observées après irradiation, reflétées d'une part par une diminution importante de la quantité de protéines totales, et d'autre part, par la chute du niveau des activités totales des différentes classes d'enzymes. Ce « potentiel» enzymatique sécrété dans le suc pancréatique diminue de façon importante à partir du $3^{\mathrm{e}}$ jour après l'exposition. 
Ce type de réponse a été rapporté quelques jours après irradiation localisée du pancréas chez le chien, avec une diminution du nombre de granules de zymogènes, représentant le stock de pro-enzymes de la cellule, et qui s"est maintenue pendant 2 à 3 semaines. D'autre part, la morphologie normale des zymogènes n'a pas été recouvrée 8 mois après irradiation, s'accompagnant d'une baisse de la concentration en enzymes dans le tissu pancréatique (Volk et Wellmann, 1968).

Du fait de la grande capacité de réserve de la sécrétion pancréatique exocrine, il est fort probable que la diminution radio-induite de la quantité d'enzymes arrivant dans la lumière duodénale, telle que nous l'avons observée dans notre étude, n'engendre pas de conséquences sévères sur la disponibilité des nutriments dans les jours qui suivent l'irradiation.

Si les conséquences à court terme des atteintes pancréatiques sont difficilement démontrables, celles à des temps plus tardifs s'envisagent plus facilement. Tout d'abord, le fait d'un renouvellement cellulaire lent, le pancréas est généralement considéré comme un organe peu sensible à l'irradiation. Certains auteurs ont même émis l'hypothèse que les variations radio-induites de la sécrétion pancréatique exocrine seraient la conséquence de dommages d'autres systèmes plus radio-sensibles (Telbisz et al., 2002). Dans le cas de lésions radio-induites sévères du tissu pancréatique, les symptômes cliniques sont rarement associés, probablement dû à la grande capacité de réserve de cet organe mais également aux conditions expérimentales non adaptées à ce type d'observation. Cependant, chez le porc, il a été montré qu'une irradiation hémicorporelle postérieure de $11 \mathrm{~Gy}$, entraîne au bout de 4 à 6 mois, des lésions pancréatiques plus ou moins intenses, mais dont les répercussions nutritionnelles sont capitales; en effet, l'atrophie pancréatique va conditionner les capacités digestives des animaux, leur consommation alimentaire et, de ce fait, leur survie (Daburon et al., 1978). Chez le patient irradié pour des raisons thérapeutiques, il est évident qu'un syndrome de malabsorption, résultant en partie d'une mauvaise digestion des nutriments par altération des fonctions pancréatiques exocrines, peut contribuer à une baisse de qualité de vie. Ainsi, la relation de cause à effet entre l'atteinte pancréatique et ses répercussions sur d'autres fonctions du tractus digestif, même à long terme, ne doit pas être négligée dans la mise en place de protocoles de radiothérapie.

En conclusion, l'irradiation corps entier de porcs à la dose de $6 \mathrm{~Gy}$ au plan médian entraîne des modifications fonctionnelle de la sécrétion pancréatique exocrine ; cette phase aiguë se traduit par une diminution précoce et importante du volume mais également de la composante enzymatique du suc pancréatique. Cependant, si l'atteinte fonctionnelle est bien réelle, son origine reste encore incertaine ; l'hypothèse d'une modulation de la régulation de la sécrétion plutôt que la conséquence directe de dommages sur les cellules sécrétrices est vraisemblable mais reste encore à démontrer. 
Enfin, si ces effets à court terme ne représentent pas des éléments majeurs pour compromettre le pronostic vital d'une personne irradiée, ils pourraient être impliqués dans des conséquences à plus long terme observées au niveau de la glande pancréatique elle-même, mais également au niveau de l'intestin.

Remerciements. Les auteurs tiennent à remercier tout particulièrement Madame Georgette Brachet, Monsieur Francis Cointepas et Monsieur Gérard Lebonnois (INRA, Jouy-en-Josas, France) pour les soins apportés aux animaux, et Monsieur Jean Louis Lefaix (CEA, Jouy-en-Josas, France) pour son aide lors des séances d'irradiation.

\section{RÉFÉRENCES}

Ahmadu-Suka F., Gillette E.L., Withrow S.J., Husted P.W., Nelson A.W., Whiteman C.E. (1988) Pathologic response of the pancreas and duodenum to experimental intraoperative irradiation, Intern. J. Radiat. Oncol. Biol. Phys. 14, $1197-1204$

Anno G.H., Baum S.J., Withers H.R., Young R.W. (1989) Symptomatology of acute radiation effects in humans after exposure to doses of 0.5-30 Gy, Health Phys. 56, 821-838.

Archambeau J.. Griem M., Harper P. (1966) The effects of $250 \mathrm{kV}$ X-rays on the dog's pancreas: morphological and functional changes, Radiat. Res. 28. 243-256.

Becciolini A., Gariaggi P., Arganini L., Castagnoli P., De Giuli G. (1974) Effect of 200, 650 and $1200 \mathrm{R}$ on the intestinal disaccharidases and dipeptidases, Acta Radiol. Ther. Phys. Biol. 13, 141-152.

Bieth J., Métais P., Warter J. (1966) Étude des protéases pancréatiques. I - Dosage de la trypsine par la benzoyl-arginine-P-nitroanilide et ses applications, Ann. Biol. Clin. 24, 787-803.

Bieth J., Métais P., Warter J. (1968) Étude des protéases pancréatiques. II - Dosage de la chymotrypsine par la succinyl-phénylalanine-P-nitroanilide et ses applications, Ann. Biol. Clin. 26, 143-158.

Bieth J., Spiess B., Wermuth C.G. (1974) The synthesis and analytical use of a highly sensitive and convenient substrate of elastase, Biochem. Med. 11, 350-357.

Bradford M. (1976) A rapid and sensitive method for the quantification of microgram quantities of protein utilizing the principle of protein-dye binding, Ann. Biochem. 72, 248-254.

Corring T., Aumaitre A., Rérat A. (1972) Fistulation permanente du pancréas exocrine chez le porc. Application : réponse de la sécrétion pancréatique au repas, Ann. Biol. Anim. Bioch. Biophys. 12, 109-124.

Corring T., Daburon F., Rémy J. (1974) Effets de l'irradiation aiguë sur la sécrétion du pancréas exocrine che\% le porc, Strahlentherapie 149, 417-425.

Daburon F., Rémy J., Nizza P. (1972) Étude du syndrome gastro-intestinal d'irradiation chez le porc, Strahlentherapie 144, 343-361.

Daburon F., Duée P.H., Tricaud Y., Bourhoven D., Marion C. (1978) Bilans nutritionnels chez le porc irradié à forte dose (1100 rd) sur la moitié postérieure du corps, Ann. Biol. Anim. Bioch. Biophys. 18(3), 65I-662.

Delaney J.P., Bonzak M. (1992) Acute radiation enteritis in rats: bile salts and trypsin, Surgery 112, $587-592$

Dubois A., Jacobus J.P., Grissom M.P., Eng R., Conklin J.J. (1984) Altered gastric emptying and prevention of radiation induced vomiting in dogs, Gastroenterology 86, 444-448.

Du Toit D.F., Heydenrych J.J., Smit B., Zuurmond T., Louw G., Laker L. (1987) The effect of ionizing radiation on the primate pancreas : an endocrine and morphologic study, J. Surg. Oncol. 34, $43-52$. 
Frankendal B. (1974) Gastrointestinal protein loss induced by ${ }^{60} \mathrm{Co}$ irradiation of the abdomen in mice. Acta Radiol. Ther: Phys. Biol. 13,65-82.

Gudkova E.A. (1957) Changes in the external secretion on the pancreas during acute radiation sickness of various degrees of severity. E\%hegodnik. IEM. AMN SSSR, Leningrad.

Gudkova E.A. (1960) Mechanisms of the influence of innizing radiation on the external secretion of the pancreas, Transactions of the scientific conlerence on problems in the physiology and pathology of digestion. Memory of Academician K. M. Bykov, pp. 21.3-219.

Hauer-Jensen M., Skjonsberg G., Moen E., Clausen O.P. (1995) Intestinal morphology and cytokinetics in pancreatic insufficiency. A experimental study in the rat, Dig. Dis. Sci. 40, 2170 2176.

Hiatt N., Werner N.E. (1967) Influence of intestinal content on radiation lesions of the small intestine, Proc. Soc. Exp. Biol. Med. 124, 937-939.

Juste C., Corring T., Le Coz Y. (1983) Bile restiution procedures for studying bile secretion in fistulated pigs, Lab. Anim. Sci. 33, 199-202.

Middleton G.R., Young R.W. (1975) Emesis in monkeys following exposure to ionizing radiation, Aliat. Space Environ. Med. 46, 170-172.

Morgenstern L., Hiatt N. (1967) Injurious effect of pancreatic secrelions on postradiation enteropathy, Gastroenterology 53, 92.3-929.

Morgenstern L., Snyder P., Krohn H.L., Hiatt N. (1970) Prolongation of survival in lethally irradiated dogs by pancreatic duct ligation, Arch. Surg. 101. 586-589

Mulholland M.W., Levitt S.H., Song C.W., Potish R.A. (1984) The role of luminal contents in radiation enteritis, Cancer 54, 2396-2402.

Palladino M.A., Galton J.E., Troll W., Thorbecks G.J. (1982) Gamma-irradiation-induced mortality: protective effect of protease inhibitors in chickens and mice, Int. J. Radiat. Biol. 41. 183-191.

Patin S., Krohn, H.L., Hiatt, N. (1970) Prolongation of survival in lethally irradiated dogs, Arch. Surg. 101, 586-589.

Rachootin S., Shapiro S., Yamakawa T., Goldman L., Patin S., Morgenstern L. (1972) Potent antiprotease derived from ascaris lumbricoides. Ellicacy in amelioration of post-radiation enteropathy, Gastroenterology 62, 796-802.

Rémy J.. Daburon F., Villiers. P.A.. Chenut M.C. (1974) Modification de l’absorption intestinale après irradiation aiquë chez le porc, Strahlentherapic 148. 95-106.

Rémy J., Daburon F., Villiers P.A., Dudedat P. (1975) Ellets de l'irradiation aiquë à dose létale sur la sécrétion gastrique chez le porc, Strahlentherapie 150, 532-5.38.

Scanff P., Grison S., Monti P., Joubert C., Grifiths N.M.. Gournclon P. (1997) Whole-body gamma irradiation modifies bile composition in the pig. Radiat. Ress, 148, 175-180).

Schaffalitzky de Muckadell O.B., Farhenkrug J.. Nielson J.. Westphall I.. Worning H. ( 981 ) Mealstimulated secretin release in man: effect of acid and bile. Scand. I. Gastroenterol. 16.981-988.

Solomon T.E. (1984) Regulation of pancreatic secretion. Clin. Gastroemerol. 13.657-678.

Thomson A.B.R., Cheesman C.I., Walker K. (1983) Effect of abdominal irradiation on the kinetic parameters of intestinal uptake of glucose, galactose. leucine and $g l y$-leucine in the rat. $J . L a b$. Clin. Med. 102, 81,3-827.

Stevens C.E. (1977) Comparative physiology of the digestive system. Duke's Phwsiology of the Domestic Animal (Swenson M.L.. Ed.) pp. 216-232. Comell University Press. New York. USA.

Telbisz A., Kovacs A.L., Somosy Z. (2002) Inlluence of X-ray on the autophagic-lysosomal system in rat pincreatic acini, Micron 33, 143-151.

Volk B.W., Wellmann K.F. (1968) The effect ol tadiation on ultrastructure and enzymes of the canine pancreas, Gastrointestinal Radiation Injar! (Sullivan M.F.. Ed.) pp. 73-93. Excerpla Medica Found. Washington, USA. 